\title{
Assessment of Factors Affecting Accuracy and Repeatability in Semi-Automated Echocardiographic Measurement of Chamber Volume Using a Physical Phantom
}

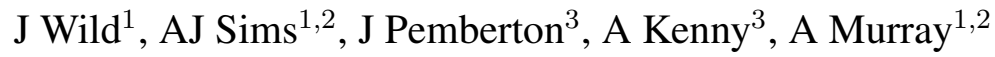 \\ ${ }^{1}$ University of Newcastle upon Tyne, Newcastle upon Tyne, UK \\ ${ }^{2}$ Regional Medical Physics Department, Freeman Hospital, Newcastle upon Tyne, UK \\ ${ }^{3}$ Department of Cardiology, Freeman Hospital, Newcastle upon Tyne, UK
}

\begin{abstract}
Three dimensional echocardiography offers the benefit of non-invasive measurement of chamber volume at the cost of increased effort of data handling. Automated or semi-automated image analysis may help to reduce manual effort but can embody assumptions and limitations which have a significant effect on results.

We used a laboratory balloon phantom to study the effect of three factors used in a semi-automated image analysis technique. These factors were: a) the use of $2 D$ or $3 D$ image gradient operators; b) manual or automatic detection of base; c) fixed or case-by-case limit of short-axis radius.

We found that $3 D$ image gradient operators were more accurate than their traditional $2 D$ counterparts; that manual identification of the base had no effect on accuracy or repeatability, and that setting the maximum short axis radius on a case-by-case basis was more accurate and repeatable than achieved with a constant value.
\end{abstract}

\section{Introduction}

Real time three-dimensional echocardiography (RT3DE) offers the prospect of rapid acquisition of volumetric images and non-invasive measurement of chamber volumes. It has been shown to provide more accurate and repeatable measures of chamber volumes over traditional 2D echocardiography $[1,2]$. However, the spatial and temporal resolutions achieved by modern ultrasound systems are associated with high data rates, and there is a need for automated, or semi-automated image analysis techniques to make measurement of chamber volume a realistic proposition. For this reason, research into automated and semiautomated image analysis to assist clinicians has been a popular area of study $[3,4]$.

Assumptions, choices and necessary manual interventions embodied within image analysis algorithms affect their repeatability and accuracy. In this study we aimed to measure, using a laboratory balloon phantom, the effects on repeatability and accuracy of a) the choice of two- or three- dimensional gradient detection kernel; b) the presence or absence of a manual intervention to define the locations of the base of the chamber; c) the choice of an individual or fixed short-axis maximum radius.

\section{Methods}

\subsection{Image acquisition}

A Philips Sonos 7500 ultrasound system with a $\times 4$ transducer was used to image a balloon filled with water. The balloon was suspended in a water bath whilst a cardiologist imaged the balloon from above. Two scans were performed sequentially for nine different volumes $(50 \mathrm{ml}, 75 \mathrm{ml}, 100 \mathrm{ml}, 150 \mathrm{ml}, 200 \mathrm{ml}, 250 \mathrm{ml}, 300 \mathrm{ml}, 400 \mathrm{ml}$, $500 \mathrm{ml})$. The balloon opening was deemed to represent the mitral valve.

\subsection{Calculation of chamber volume}

The volume of fluid in each balloon was calculated using a semi-automated technique [5], illustrated in figure 1. One point near the apex and one near the base of the balloon were selected manually to define the long axis. A series of perpendicular contiguous planes along the long axis were subsequently extracted as $2 \mathrm{D}$ short-axis images from the 3D image and subjected to image gradient magnitude detection, thresholding, and morphological erosion. This process resulted in binary images upon which circular borders were fitted. The apical plane circle was fixed with a radius of 1 pixel and, by definition, located on the long axis.

Circular fits to short axis wall boundaries were rejected if their radius exceeded a fixed upper limit, or if their location in the short axis plane deviated from that of their 


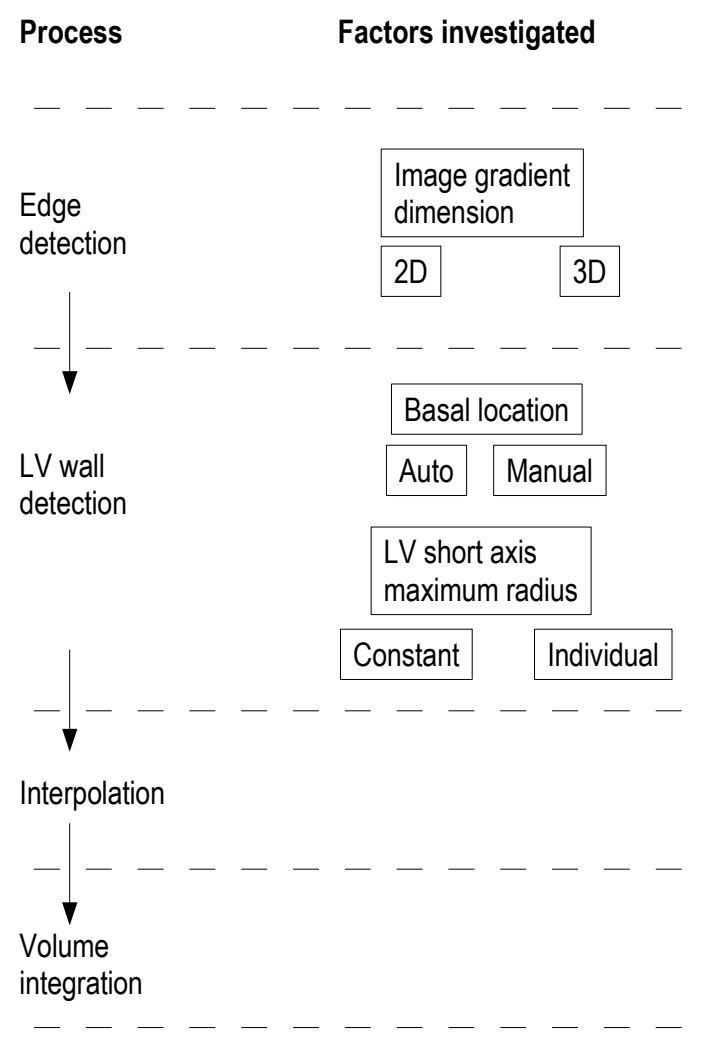

Figure 1. Flowchart showing the volume calculation process and the factors for which the effect of variation was investigated.

neighbours by more than a pre-defined limit. Rejected boundaries were replaced by circular boundaries which were linearly interpolated from the nearest pair of accepted boundaries on either side. Balloon volumes were calculated by integration of planar boundary area along the long axis.

\subsection{Factors investigated}

Three different factors were investigated for their effect on repeatability and accuracy of calculated volume. These were: a) the dimensionality of image gradient magnitude operator (2D or $3 \mathrm{D})$; $b$ ) the presence or absence of manual specification of the basal circle; c) the application of either a constant or individual maximum radius for the short-axis boundary. Figure 1 shows the relative order of application of these factors in the volume calculation process.

Each of the three factors had two possible values. Balloon volumes were calculated for all $8(2 \times 2 \times 2)$ possible combinations of factor values.

\begin{tabular}{lllll}
\hline $\begin{array}{l}\text { Image } \\
\text { Gradient }\end{array}$ & Basal & Maximum & $\begin{array}{l}\text { Bias } \\
(\%)\end{array}$ & $\begin{array}{l}\text { Standard } \\
\text { Deviation (\%) }\end{array}$ \\
\hline 2D & Not Set & Fixed & $12 \%$ & $16 \%$ \\
2D & Not Set & Individual & $-5 \%$ & $12 \%$ \\
2D & Set & Fixed & $-11 \%$ & $16 \%$ \\
2D & Set & Individual & $-4 \%$ & $11 \%$ \\
3D & Not Set & Fixed & $-7 \%$ & $17 \%$ \\
3D & Not Set & Individual & $2 \%$ & $11 \%$ \\
3D & Set & Fixed & $-8 \%$ & $17 \%$ \\
3D & Set & Individual & $-1 \%$ & $13 \%$ \\
\hline
\end{tabular}

Table 1. Summary of repeatability results for eight combinations of image analysis methods applied to 9 balloon phantoms $(50 \mathrm{ml}, 75 \mathrm{ml}, 100 \mathrm{ml}, 150 \mathrm{ml}, 200 \mathrm{ml}, 250 \mathrm{ml}$, $300 \mathrm{ml}, 400 \mathrm{ml}, 500 \mathrm{ml})$.

\begin{tabular}{|c|c|c|c|c|}
\hline Image & Basal & Maximum & Bias & Standard \\
\hline Gradient & Location & Radius & $(\%)$ & Deviation (\%) \\
\hline $2 \mathrm{D}$ & Not Set & Fixed & $18 \%$ & $15 \%$ \\
\hline 2D & Not Set & Individual & $4 \%$ & $10 \%$ \\
\hline 2D & Set & Fixed & $17 \%$ & $16 \%$ \\
\hline 2D & Set & Individual & $4 \%$ & $10 \%$ \\
\hline 3D & Not Set & Fixed & $9 \%$ & $15 \%$ \\
\hline 3D & Not Set & Individual & $1 \%$ & $10 \%$ \\
\hline $3 \mathrm{D}$ & Set & Fixed & $8 \%$ & $15 \%$ \\
\hline $3 \mathrm{D}$ & Set & Individual & $0 \%$ & $9 \%$ \\
\hline
\end{tabular}

Table 2. Summary of accuracy results for eight combinations of image analysis methods applied to 9 balloon phantoms $(50 \mathrm{ml}, 75 \mathrm{ml}, 100 \mathrm{ml}, 150 \mathrm{ml}, 200 \mathrm{ml}, 250 \mathrm{ml}, 300 \mathrm{ml}$, $400 \mathrm{ml}, 500 \mathrm{ml})$. Bias is known - calculated volume.

\subsection{Method of comparision}

The method of Bland and Altman [6] was used to calculate repeatability (by comparing repeat scans), and accuracy (by comparing mean calculated volume with known volume). A generalised linear ANOVA was used to detect the factors that affected repeatability and accuracy. Percentage volume differences (\%) were used in the analysis rather than actual volume differences $(\mathrm{ml})$ to account for the effect of the large range of balloon volumes.

\section{Results}

Results for repeatability (comparisons of calculated volume for repeat scans) are shown in table 1. Results for accuracy (comparison of calculated volume with known volume) are shown in table 2 .

The choice of image gradient dimension had no significant effect on the repeatability, but was found to have a significant effect on accuracy $(P=0.003)$. Application of a 3D gradient kernel improved the accuracy. Setting the location 


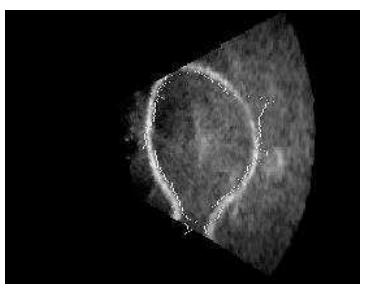

(a) $100 \mathrm{ml}$ original scan

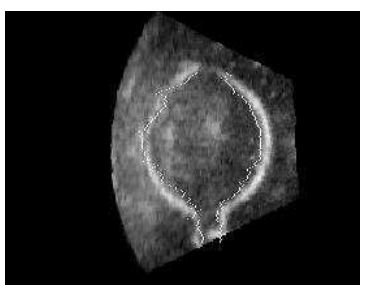

(c) $250 \mathrm{ml}$ original scan
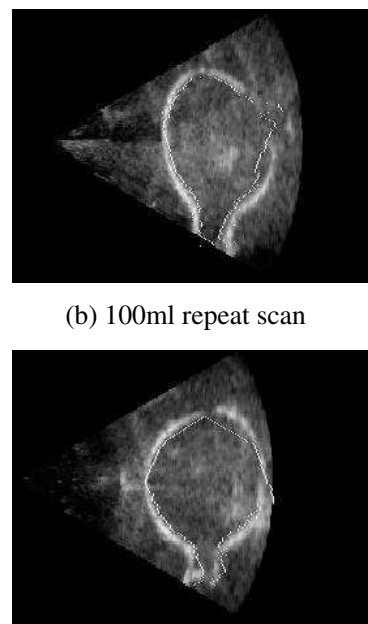

(d) $250 \mathrm{ml}$ repeat scan (b) $100 \mathrm{ml}$ repeat scan

Figure 2. Long-axis images for $100 \mathrm{ml}$ and $250 \mathrm{ml}$ balloons with detected wall shown in white.

of the basal circle manually was found to have no significant effect on either repeatability or accuracy for these images. Application of a value for the maximum short axis radius which was chosen for each scan was found to significantly improve both repeatability $(P=0.011)$ and accuracy $(P<0.001)$ when compared with those calculated using a constant radius.

The best combination of factor choices was: 3D image gradient combined with setting a per-scan maximum shortaxis radius. This gave a mean percentage difference between known and calculated volumes of $0.2 \%$ (95\% confidence interval of $-6 \%$ to $+7 \%)$, or $2 \mathrm{ml}(95 \% \mathrm{CI}$ of -19 to $+15 \mathrm{ml}$ ) for absolute volume difference. Figure 2 shows long axis images for $100 \mathrm{ml}$ and $250 \mathrm{ml}$ balloons with the detected wall overlaid in white.

\section{Discussion and conclusions}

We found that using 3D image gradient operators gave better accuracy than using 2D operators for the calculation of chamber volume compared with known volumes. We did not detect a difference in repeatability when comparing the two types of image gradient operator. Figure 3 shows an example in which the $3 \mathrm{D}$ version performed better at detecting a weaker boundary (on the left side of the images) than the $2 \mathrm{D}$ version. Three dimensional image gradient operators are sensitive to changes in pixel intensity due to surfaces aligned in any direction; they are therefore less susceptible to poorer signal to noise ratio in any two dimensional plane than their 2D couterparts.

We did not detect any effect on accuracy or repeatability of calculation of volume due to the method of locating the base of the chamber. Principally, the relatively small

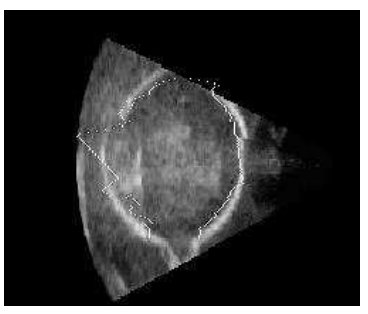

(a) 2D Image gradient

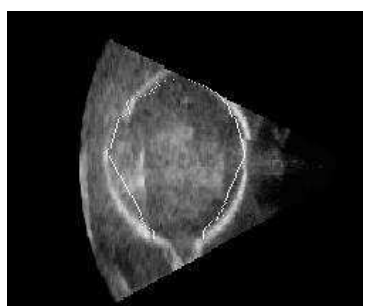

(c) 3D Image gradient

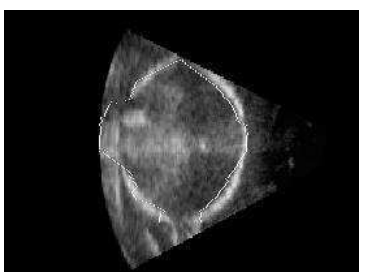

(b) 2D Image gradient

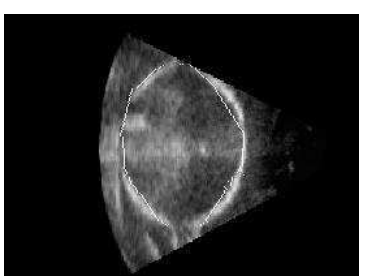

(d) 3D Image gradient
Figure 3. Effect of image gradient. Long axis views with the LV wall superimposed in white are shown. An original and repeat scan are shown side by side. It can be seen that there is little difference between repeat scans. However, the LV wall detection is improved by increasing the dimensionality of image gradient used.

volume in the basal area of the balloon did not have much influence on the total volume, as illustrated by figures 4(c) and $4(d)$. Secondly, if circles near to the base were incorrectly accepted, as in figure 4(e), setting the base location had a limited effect on volume because the intermediate circles were interpolated between the base and the incorrectly accepted circles as in figure 4(f). For most balloons there was an accepted circle within a relatively short distance from the base.

With consideration to clinical images, the corresponding basal shape of the balloon is dissimilar to normal and abnormal left ventricles in which the mitral valve diameter is wider and relatively more similar to the maximum left ventricle diameter. Our results for this factor may not be applicable to clinical studies.

No attempt was made by the operator to standardise the field of view occupied by the balloon images; the main concern was to achieve the best image quality. An example is shown in figure 5. These two balloons are the smallest and largest in relation to image size of all the balloon images. It can be seen with the smaller balloon 5(a) that a fixed circle containment value allowed several larger circles to be accepted which skewed the total volume. Using an individual radius avoided this problem. This is analagous to the clinical situation in which there is significant variability in the way different cardiologists image patients in respect to image size (magnification), and sig- 


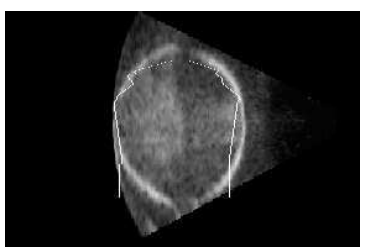

(a) Mitral not set

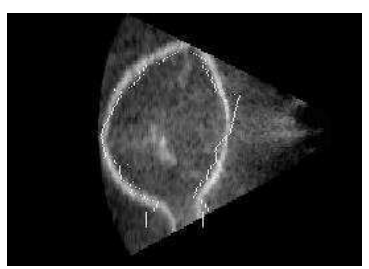

(c) Mitral not set

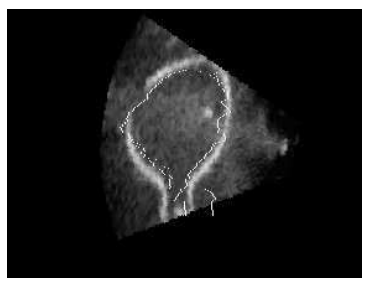

(e) Mitral not set
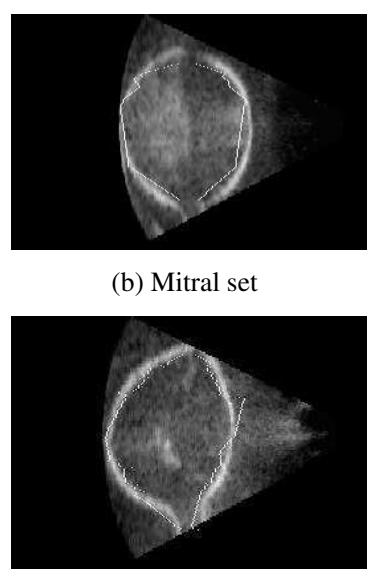

(d) Mitral set

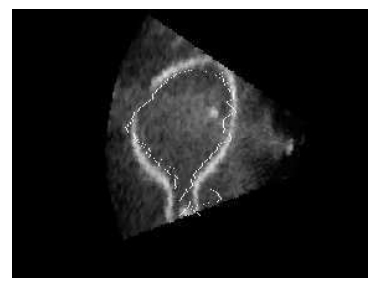

(f) Mitral set (b) Mitral set

Figure 4. Effect of setting the basal location (balloon opening). Six long axis images are shown before and after setting the basal location. In (a) and (b) there is a significant difference in volume. In (c) and (d) the relative small volume of the basal area does not influence the overall volume difference. In (e) and (f) incorrectly accepted circles near the base limit the difference in volume.

nificant variation in the natural size of ventricles between patients. We have shown that setting the maximum shortaxis radius on a case-by-case basis gave more accurate and more repeatable results than by setting a fixed value for all cases.

In this study, we have shown that accuracy was improved by use of 3D image gradient operators and by application of an individual maximum radius for the short axis. 3D image gradient operators improved the signal to noise ratio, and hence helped border fitting by reducing the effect of false edges arising from noise. Setting a maximum short-axis radius was necessary to ensure that borders were not fitted to noisy pixels outside the chamber wall; greater accuracy and repeatability was achieved when this value was set on a case-by-case basis. Manual identification of basal location was found to be unnecessary for an ideal phantom, but may be required in clinical images.

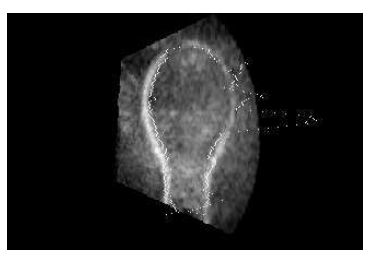

(a) Fixed

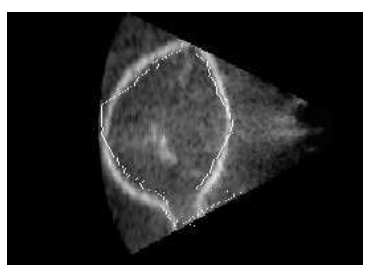

(c) Fixed
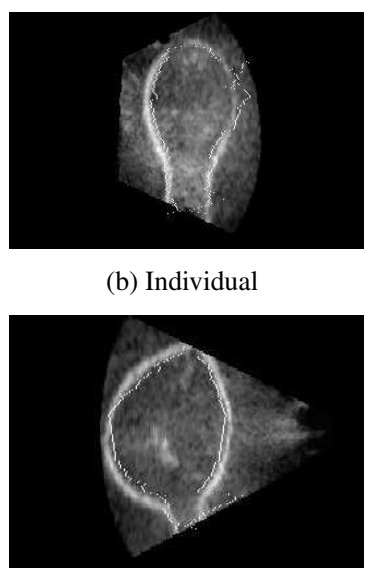

(d) Individual (b) Individual

Figure 5. Two long axis images showing the effect of fixed and individually set maximum short-axis for two balloons with different image sizes.

\section{References}

[1] Weyman A. The year in echocardiography. Journal of the American College of Cardiology 2006;47:856-863.

[2] Sugeng L, Weinert L, Lang R. Left ventricular assessment using real time three dimensional echocardiography. Heart 2003;89:iii29-iii36.

[3] Noble J. Ultrasound image segmentation: a survey. IEEE Transactions on Medical Imaging 2006;25:987-1010.

[4] Frangi AF, Niessen WJ, Viergever MA. Three-dimensional modeling for functional analysis of cardiac images: a review. IEEE Transactions on Medical Imaging 2001;20:2-25.

[5] Wild J, Sims A, Pemberton J, Irvine T, Kenny A, Murray A. Calculation of left ventricular volume from 3D echocardiography: Comparison of three image analysis techniques. Computers in Cardiology 2004;31:61-64.

[6] Bland J, Altman D. Statistical methods for assessing agreement between two methods of clinical measurement. Lancet 1986;1:307-310.

Address for correspondence:

Dr Andrew J. Sims

Regional Medical Physics Department,

Freeman Hospital,

Newcastle upon Tyne,

NE7 7DN,

UK.

a.j.sims@newcastle.ac.uk 